\title{
Muscle pathology in idiopathic cricopharyngeal dysphagia
}

\section{Enzyme histochemical and electron microscopic findings}

\author{
E. Laurikainen $^{1}$, K. Aitasalo ${ }^{1}$, P. Halonen ${ }^{3}$, B.Falck ${ }^{4}$, and H.Kalimo ${ }^{2}$ \\ Departments of ${ }^{1}$ Otorhinolaryngology, ${ }^{2}$ Pathology, ${ }^{3}$ Neurology and ${ }^{4}$ Clinical Neurophysiology, \\ The University of Turku and Turku University Central Hospital, Turku, Finland
}

Received December 18, 1991 / Accepted December 27, 1991

\begin{abstract}
Summary. The structural changes in the cricopharyngeal muscle $(\mathrm{CM})$ were examined ultrastructurally and by enzyme histochemistry in five patients suffering from idiopathic cricopharyngeal dysphagia (ICD). Diagnosis was established by fiberoptic esophagoscopy, esophageal manometry and cineradiography. Cricopharyngeal myotomy was performed with marked improvement in all patients. Intraoperatively, a biopsy was taken from the $\mathrm{CM}$. Additionally, all patients underwent neurological examination for possible generalized muscle disease, and a biopsy was taken from a limb muscle. CM from nine cadavers without known history of dysphagia served as control. The control samples disclosed structural changes which were considered to be pathological in other skeletal muscles, and required that the criteria for $\mathrm{CM}$ pathology we modified accordingly. In three patients changes in CM histology suggested specific pathogenesis: one patient had evidence for a generalized myositis but was only symptomatic for dysphagia. Another patient had muscle fiber atrophy and slight inflammation in her CM, possibly due to alcohol abuse. The third patient had loss of $\mathrm{CM}$ fibers with replacement by connective tissue enough to cause functional disturbances. In two patients no cause for dysphagia was found in either immunohistochemistry or electron microscopic studies. These results demonstrate the special structural features of the CM and indicate that ICD can have multiple etiologies.
\end{abstract}

Key words. Cricopharyngeal dysphagia - Muscle biopsy Electron microscopy - Enzmye histochemistry - Myositis

\section{Introduction}

Dysphagia is a common, disabling symptom occurring in a variety of different diseases, e.g. postoperative or posttraumatic paresis of the caudal cranial nerves, brain-

Correspondence to: E. Laurikainen, Kresge Hearing Research Institute, Department of Otolaryngology, The University of Michigan, 1301 East Ann Street, Ann Arbor, MI 48109, USA stem strokes, inflammatory myopathies, motor neuron disease, muscular dystrophies or dermatomyositis $[1,12$, 20, 21].

Although most often the dysphagic disorders are idiopathic, it has been suggested that idiopathic cricopharyngeal muscle (CM) dysfunction (ICD) causes hypertrophy of the CM, followed by incoordinated initiation of swallowing, nasopharyngeal reflux, hoarseness, weight loss and asthma. An elevated intraluminal pressure against a closed CM can also result in outpouching of esophageal mucosa (Zenker's diverticulum) in a specific area of physiological weakness $[6,15]$.

In esophageal manometric and cineradiographic studies of patients with ICD the major findings have been epiglottic dysmotility, defective closure of the laryngeal vestibule, paresis in pharyngeal constrictors, incoordinated relaxation of the CM, Webs and Zenker's diverticulum $[8-11,19,22]$. However, only a few studies have been published on the pathology of ICD. Cruse et al. [5] found severe fibrosis in the CM and concluded that dysphagia was secondary to muscle fiber damage, but the primary cause remained unknown. Hanna and Henderson [13] found marked ultrastructural changes: numerous and aberrant mitochondria, increased glycogen, lipid inclusions, phagolysosomes and nemaline rods. The interpretation of these findings must be re-evaluated, in the light of recent studies $[3,17]$ showing that most of these changes also appear in control CMS.

In the present study we have analyzed the CMs from five patients with severe ICD using enzyme and immunohistochemistry and electron microscopy (EM). Dysfunction of the CM was confirmed by fiberoptic esophagoscopy, esophageal manometry and cineradiography of the pharyngoesophageal segment. Muscle biopsies were taken from the CM during planned myotomy $[11,14,17,25]$. Criteria for an unaffected $\mathrm{CM}$ were based on control samples taken from nine cadavers (ranging in age from a fetus of 22 weeks up to a decedent who was 81 years old). None had a preceding history of swallowing disorders. Our goal was to compare clinically observed functional findings to laboratory results in ICD patients. A special effort was directed to comparison of the ultra- 
structural features of $\mathrm{CM}$ in the ICD patients and the cadaver controls.

\section{Patients and methods}

$\mathrm{CM}$ myotomies were performed in patients with clear clinical signs of $\mathrm{CM}$ achalasia, pouch formation or a positive vallecula sign in cineradiography together with an elevated resistance for an endoscope or elevated pressure at the sphincter level recorded with manometry. Any patient with persistent central nervous system disease, other known muscular disorders, infection, trauma or known etiology for a swallowing problem was excluded. The characteristics of the five patients who fulfilled the criteria and underwent operation are presented in Table 1.

\section{Operative technique}

The procedure for $\mathrm{CM}$ myotomy was carried out according to the technique described by Henderson and Marryat [14]. A transversely cut slice of the CM was taken for histochemical and EM studies. Oral fluids were started on the 1st postoperative day after myotomy or were delayed to the $3 \mathrm{rd}$ postoperative day if diverticulum resection was performed together with myotomy.

\section{Neurological examination}

All patients underwent a thorough neurological examination 1-2 months after CM myotomy. Following this examination, electromyography (EMG) was recorded from an upper extremity and a biopsy was taken from the deltoid or vastus laterialis muscle.

\section{Control muscle samples}

Tissue samples from the control CMs were obtained during the autopsies of nine cadavers after sudden death or following a short illness without known disease that could significantly affect the $\mathrm{CM}$. All bodies were stored at $0 \pm 4^{\circ} \mathrm{C}$, with the autopsy performed within $24 \mathrm{~h}$ after death. Under these conditions the postmortem changes in the skeletal muscle structures were minimal, as previously described $[4,24]$. Data on the control subjects are given in Table 2.

Table 1. The history characteristics of the five patients suffering from idiopathic cricopharyngeal dyshagia

\begin{tabular}{|c|c|c|c|c|c|c|c|}
\hline لِّ. & 芯 & 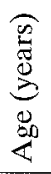 & $\begin{array}{l}\text { 峁 } \\
\text { 芯 } \\
\text { 芯 }\end{array}$ & 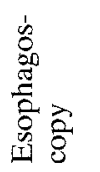 & $\frac{8}{8}$ & 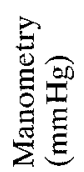 & $\frac{\vec{d}}{\stackrel{5}{0}}$ \\
\hline 1 & $\mathrm{M}$ & 70 & 1,3 & +++ & +++ & $\mathrm{N}$ & - \\
\hline 2 & F & 47 & 1 & - & + & 80 & $\mathrm{AA}$ \\
\hline 3 & M & 81 & $1-4$ & $+++^{\mathrm{a}}$ & $+^{a}$ & $\mathrm{~N}^{\mathrm{b}}$ & - \\
\hline 4 & $\mathrm{~F}$ & 80 & $1-4$ & - & ++ & $\mathrm{N}$ & Stroke \\
\hline 5 & $\mathrm{~F}$ & 66 & 1 & $++^{\mathrm{a}}$ & $+t^{\mathrm{a}}$ & $\mathrm{N}$ & - \\
\hline
\end{tabular}

$N$, The normal limits for resting pressure at cricopharyngeal level (40-60 mmHg). Symptoms 1-4: 1, subjective sensation; 2, objective swallowing difficulties; 3 , aspiration; 4 , weight loss. Resistance to passage of the fiberoptic esophagoscope is scored weak $(+)$, moderate $(++)$, and strong $(+++)$. Videofluoroscopy findings (video) at the $\mathrm{C} 4-6$ level is scored as minor $(+)$, moderate $(++)$, and strong $(+++)$; AA, alcohol abuse

a Zenker's diverticulum

b Irregular contractions in the upper third of the esophagus

c Stroke 10 years before, but with complete recovery
Table 2. Premortem findings in the nine control subjects without dysphagic signs

\begin{tabular}{llll}
\hline Patient & Sex & $\begin{array}{l}\text { Age } \\
\text { (years) }\end{array}$ & Cause of death \\
\hline 1 & M & 31 & Cerebral contusion \\
2 & M & 42 & Acute myocardial infarction \\
3 & F & 58 & Thrombosis of mesenteral artery \\
4 & F & 87 & Intracerebral hemorrhage \\
5 & M & 54 & Meningitis \\
6 & M & 78 & Acute myocardial infarction \\
7 & M & 20 & Cerebral contusion \\
8 & M & 6 & Cerebral contusion \\
9 & M & a & Respiratory distress \\
\hline
\end{tabular}

${ }^{a}$ Fetus, 22 weeks

\section{Microscopy}

The muscle biopsies taken were frozen immediately in liquid nitrogen chilled freon and stored at $-70^{\circ} \mathrm{C}$ until further processed. Frozen sections were cut in a cryostat and stained with hematoxylin and eosin (H\&E) or Gomori's trichrome. Enzyme histochemistry was done for ATPase (preincubation at pH 10.4, 4.6 and 4.3) and NADH-tetrazolium reductase (NADH-TR) [7]. Tissue sections were analyzed quantitatively using microprojection on a digitizer tablet connected with a microcomputer. For EM, small samples were fixed in 3\% phosphate-buffered glutaraldehyde, postosmicated, dehydrated in ethanol and embedded in epon. Semithin sections were stained with toluidine blue and thin sections were double-stained with uranyl acetate and lead citrate in an LKB Ultrastainer. Sections were then examined under a Jeol JEM 100C EM.

\section{Results}

The operative results and findings from five ICD patients are summarized in Table 3. Peroperatively observed in-

Table 3. Postoperative results of the five patients who unterwent operation for idiopathic criocopharyngeal dysphagia

\begin{tabular}{|c|c|c|c|c|c|c|c|}
\hline 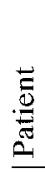 & $\stackrel{凶}{\mathscr{D}}$ & 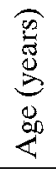 & $\frac{\mathscr{n}}{\mathrm{E}}$ & 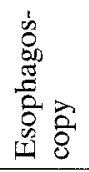 & $\frac{8}{2}$ & 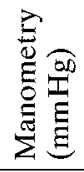 & $\frac{\vec{D}}{\overline{0}}$ \\
\hline 1 & $\mathrm{M}$ & 70 & 1,3 & - & + & $\mathrm{N}$ & $-{ }^{a}$ \\
\hline 2 & $\mathrm{~F}$ & 47 & - & - & - & $\mathrm{N}$ & $-b$ \\
\hline 3 & $\mathrm{M}$ & 81 & - & - & + & $\mathrm{N}^{\mathrm{c}}$ & -0 \\
\hline 4 & $\mathrm{~F}$ & 80 & - & - & - & $\mathrm{N}$ & - \\
\hline 5 & $\mathrm{~F}$ & 66 & - & - & - & $\mathrm{N}$ & $-^{e}$ \\
\hline
\end{tabular}

$N$, The normal limits for resting pressure at cricopharyngeal level (40-60 mmHg). Symptoms 1-4: 1, subjective sensation; 2, objective swallowing difficulties; 3 , aspiration; 4 , weight loss. Resistance to passage of the fiberoptic esophagoscope is scored weak $(+)$, moderate $(++)$, and strong $(+++)$. Videofluoroscopy findings (video) at the C4-6 level is scored as minor $(+)$, moderate $(++)$, and strong $(+++)$; Other observations:

a Slight mental deterioration

b Ulnar nerve entrapment of the right upper extremity

c Irregurlar contractions at the upper third region of esophagus

d Cachectic, weak reflexes, no specific EMG findings

e Diverticulum 
terindividual variation in $\mathrm{CM}$ thickness was large, showing no correlation between the preoperatively recorded findings and/or symptoms. Four of the five patients benefited both subjectively and objectively from the operation. Although one patient still had subjective difficulties in swallowing, all swallowing was easier than before operation and radiological and manometric examinations appeared normal. None of the patients showed signs of generalized neurological disease during neurological testing.

\section{Control muscle biopsies}

Because structural changes generally considered abnormal were frequently seen in the control CM samples, separate control biopsies from the whole age range were collected for comparison. Data of these findings are given in Table 4. The cadavers chosen for controls all died within $24 \mathrm{~h}$ of taking the $\mathrm{CM}$ samples. This time margin is acceptable for skeletal muscle EM and immunohistochemistry studies since no major changes occur in muscle structures during this period due to death $[4,24]$. The mean diameter of $\mathrm{CM}$ fibers in all age groups was about $40 \%$ smaller than the values given as normal for other skeletal muscles at the same age [7]. Furthermore, their size variation was greater than described in skeletal muscles, although there was a slight type 1 fiber predominance in all samples examined.

Marked structural changes were found in all of the control CMs (Fig. 1a, b), even in the 6-year-old body. Fiber splitting, internal nuclei and subsarcolemmal NADH-
TR-positive clumps were observed. In two controls a few lymphoid cells were seen between muscle fibers. The amount of endomysial fibrous tissue increased with age and in all controls was markedly greater than generally seen in skeletal muscles (cf. Table 4).

\section{Muscle biopsies from the ICD patients}

Patient 1. In the CM the number, distribution, size and size variation of both type 1 and 2 fibers and amount of connective tissue corresponded to those in the controls (Tables 4,5 ). In addition, there were many necrotic fibers, usually undergoing phagocytosis (Fig. 2). Many inflammatory cell infiltrates were observed, predominantly in the endomysium. Minor changes in the internal structure were encountered and only a few moth-eaten or labulated fibers were found. EM revealed abundant lipofuscin, a few necrotic fibers and inflammatory cells, but no inflammatory inclusions (undulating tubules or filaments) were detected.

In the vastus lateralis muscle biopsy (Table 5) the size variation of both fiber types was increased, atrophic fibers (mainly type 2) were frequent and pyknotic nuclear clumps were also observed. No fiber necrosis was seen, but a few split fibers and occasional lobulated fibers were found. One perivascular lymphocyte infiltrate was identified in the perimysium and a few scattered lymphocytes were noted in the endomysium (Fig. 3a, b).

Patient 2. Both CM fiber types were smaller than in the controls (Table 5). A few non-necrotic fibers were sur-

Table 4. Cricopharyngeal muscle findings of nine cadavers

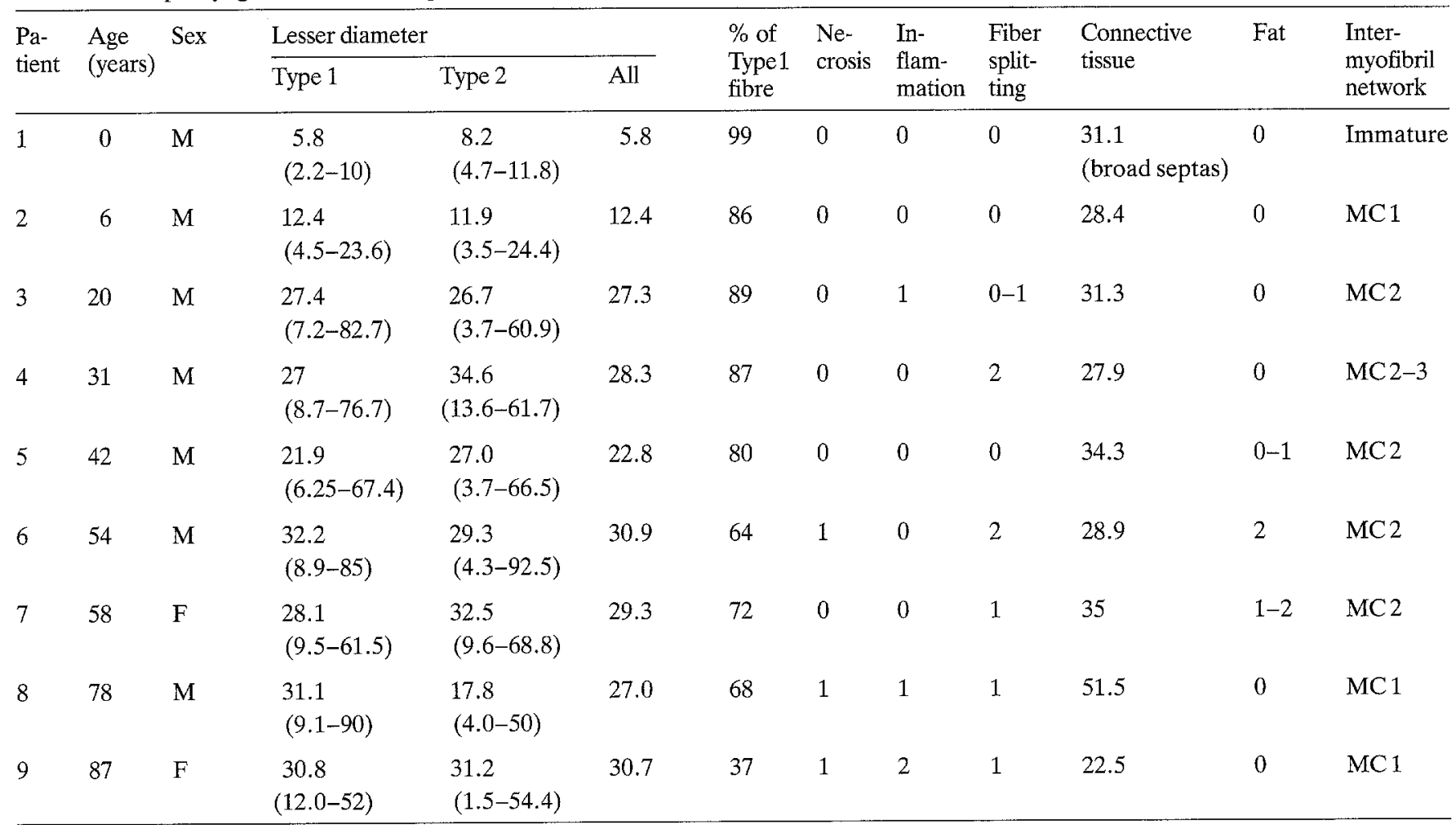

The intensity of changes is scored semiquantitatively from 0 to 3. $M C$, Mitochondrial clumps 
Table 5. Cricopharyngeal and skeletal limb muscle findings in the five patients who underwent operation for idiopathic cricopharyngeal dysphagia

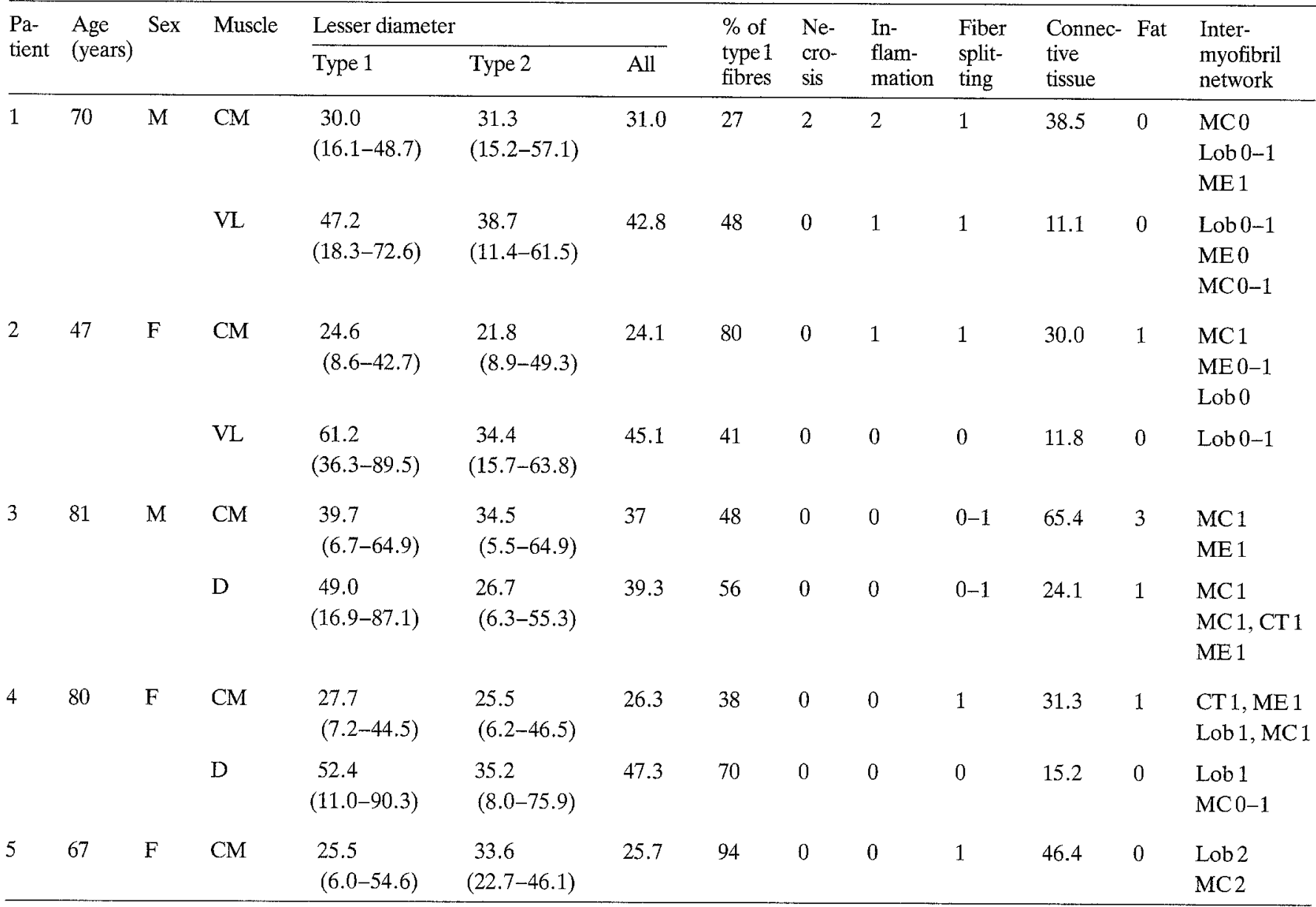

The intensity of changes is scored semiquantitatively from 0 to 3. $C M$, Cricopharyngeal muscle; $V L$, vastus lateralis muscle; $D$, deltoid muscle; $M C$, mitochondrial clumps; $L o b$, lobulated fibers; $C T$, core-targetoid fibers; $M E$, mouth-eaten fibers

rounded by lymphocytes, but necrosis or phagocytosis was not observed. Many basophilic fibers with enlarged nuclei were found. Endomysial connective tissue was abundant and fat had also accumulated between fascicles. The intermyofibrillary network was irregular and there were strongly NADH-TR-positive subsarcolemmal clumps (Fig. 4a), which in EM were verified as collections of mitochondria (Fig. 4b). Additionally, disorganized myofibrils, Z-line streaming and nemaline bodies were detected (Fig. 4c).

Vastus lateralis muscle biopsy (Table 5) showed increased size variation with slight hypertrophy of type 1 fibers and atrophy of type 2 fibers. No inflammation or other additional abnormalities were detected.

Patient 3. The CM biopsy revealed larger myofibers than in most controls (Table 5). No necrosis or inflammation was observed, but endomysial connective tissue was most abundant of all CM samples and a fair amount of fat had accumulated between the fibers. A few granular or basophilic fibers were detected and the intermyofibrillary network was often coarse or lobulated (Fig. 5).

The deltoid muscle biopsy disclosed marked fiber atrophy, predominantly of the type 2 fibers and occasion- ally occurring in small groups. Definite fiber type grouping was also seen. No necrosis or inflammation was seen but slight endomysial fibrosis and focal fat accumulation were observed.

Patient 4. In the CM biopsy muscle fibers were slightly smaller than in most controls (Table 5). No necrosis or inflammation was seen, nor was the amount of endomysial connective tissue more abundant than in most controls. Core-targetoid, moth-eaten and lobulated fibers were common, as were subsarcolemmal clumps. These clumps on EM were again identified as collections of mitochondria, a few of which contained paracrystalline inclusions (Fig. 6).

The deltoid muscle biopsy showed marked variation of fiber size and atrophy of mainly type 2. Mild fiber type grouping and a few lobulated fibers were also detected.

Patient 5. The CM fibers showed abundant mitochondrial clumps and lobulation (Table 5). The amount of connective tissue was greater than in the controls, but no signs of necrosis or inflammation were present. The vastus lateralis biopsy in this patient did not reveal any underlying general reason for the CM findings. 

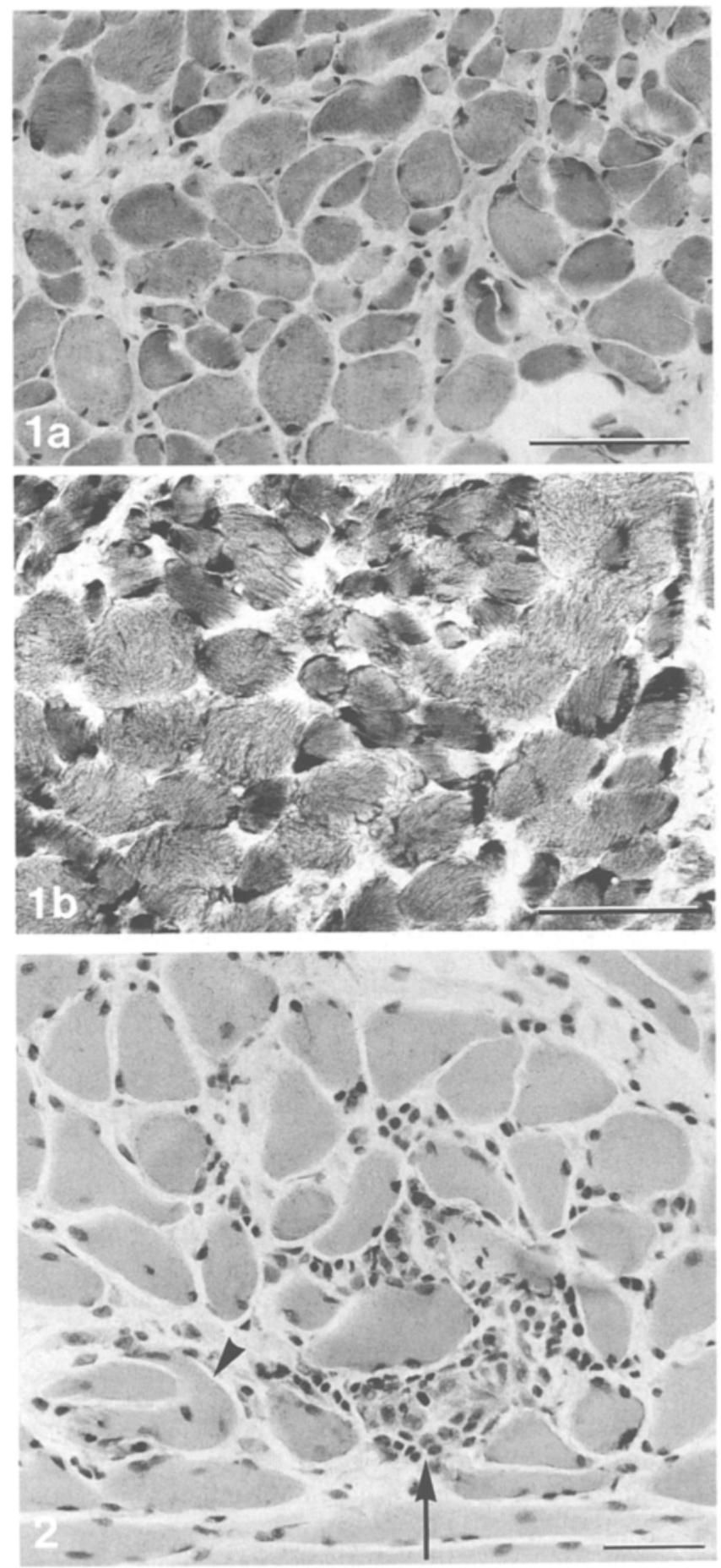

\section{Discussion}

In previous studies on CMs in patients with dysphagia the structural changes observed by Cruse et al. [5] and the mitochondrial abnormalities, phagosomes and nemaline rods found by Hanna and Hendersson [13] were considered pathological. These conclusions were based on comparisons with results from fairly limited analyses of control samples. The recent paper by Bonington's group [3] and its complementary paper by Kristmunsdottir et al. [17] have, however, demonstrated that the $\mathrm{CM}$ is an exceptional muscle.
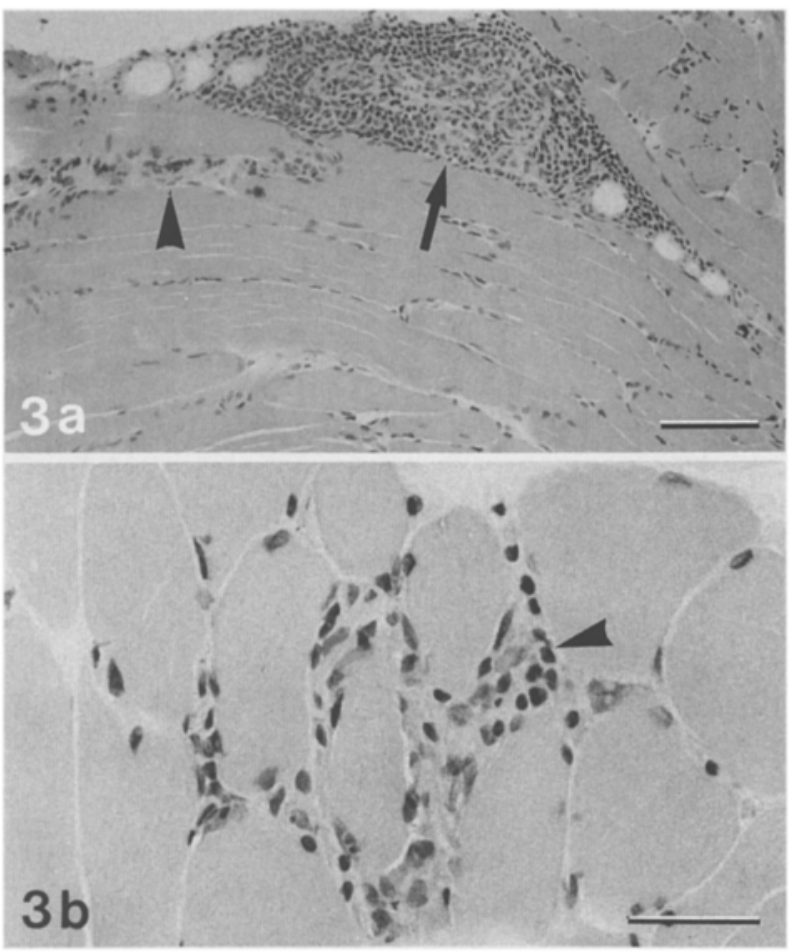

Fig.1. a Cricopharyngeus muscle from control subject 3 . The amount of endomysial connective tissue is similarly increased in the patients with idiopathic cericopharyngeal dysphagia (cf. Fig. 2): van Gieson stain: bar, $100 \mu \mathrm{m}$. b Many fibers have darkly stained subsarcolemmal clumps, as also seen in the patients (cf. Fig. 4A): NADH-TR; bar, $50 \mu \mathrm{m}$

Fig. 2. In cricopharyngeus muscle biopsy from patient 1 , one fiber (arrow) is undergoing necrosis and phagocytosis and there are inflammatory cells around adjacent fibers. The amount of connective tissue in the endomysium between the fibers is increased compared to that in the vastus lateralis muscle (cf. Fig. 3). Fiber splitting is also evident (arrowhead). H\&E; bar, $50 \mu \mathrm{m}$

Fig. 3a,b. In the vastus lateralis muscle biopsy from patient 1 , an abundant infiltrate of inflammatory cells is seen in the perimysium (arrow) with a few inflammatory cells also in the endomysium (arrowheads). Note the tightly apposed myofibers with very narrow endomysial space. $\mathrm{H} \& \mathrm{E} ; \mathbf{a}$ bar, $100 \mu \mathrm{m} ; \mathbf{b}$ bar, $50 \mu \mathrm{m}$

Our present study has shown that the size variation of $\mathrm{CM}$ fibers from fresh postmortem specimens is markedly greater than in other skeletal muscles, even at very young age, and their average size in adults is about $40 \%$ smaller than in normal skeletal muscles. Furthermore, even in subjects without dysphagia, marked degenerative changes like fiber-splitting, abnormal mitochondrial collections and fibrosis were common in the postmortem tissues studied. The amount of connective tissue of $\mathrm{CM}$ in our patients was clearly greater (average $32.3 \%$ ) than that of limb muscles (average $15.6 \%$ ) or previously published control values $(10.8 \%$ for young and $17.6 \%$ for 

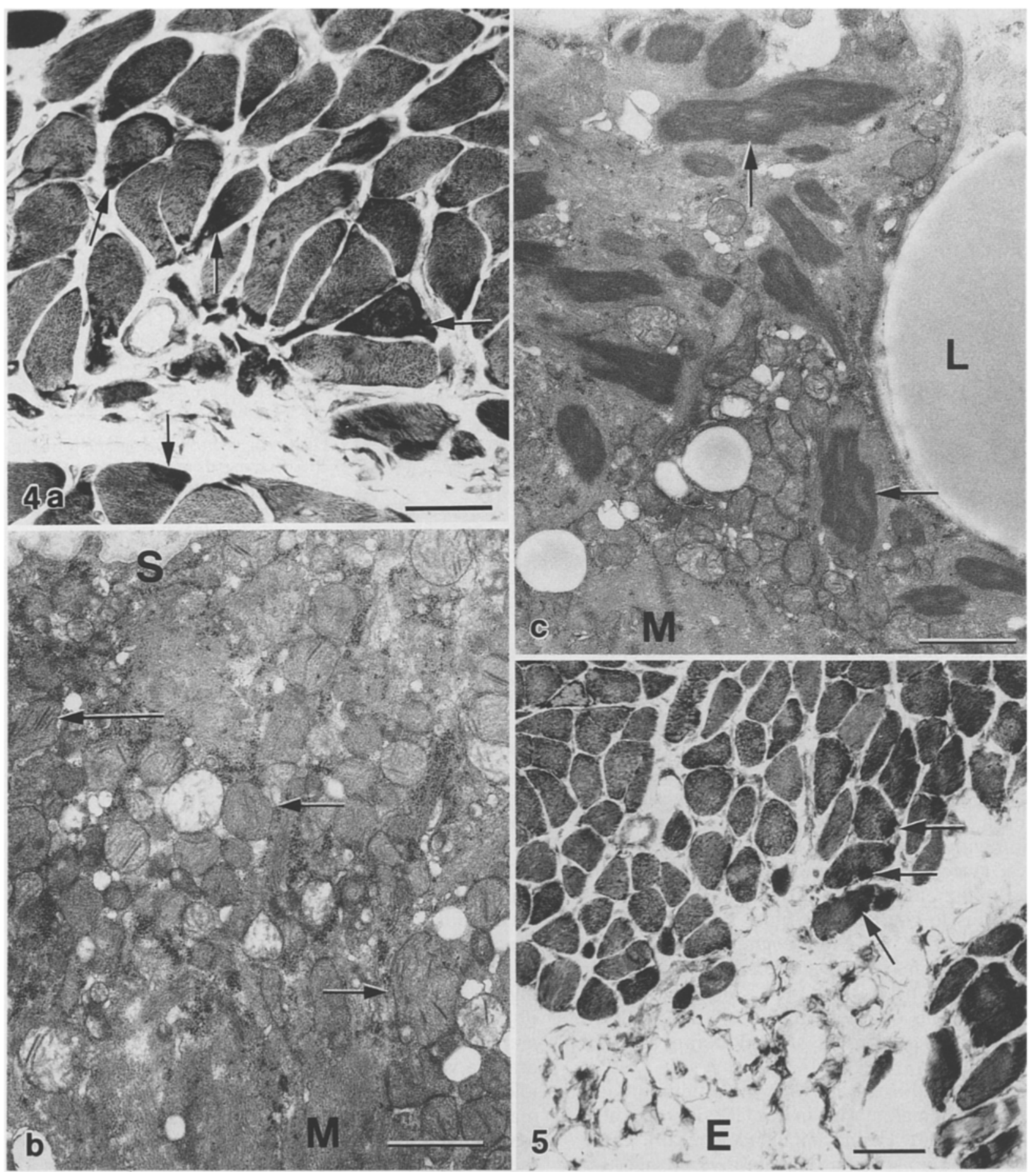

Fig. 4a-c. Cricopharyngeus muscle from patient 2. a Many fibers contain strongly stained clumps underneath their sarcolemma (arrows). NADH-TR; bar, $50 \mu \mathrm{m}$. b Electron microscopically the clumps can be defined as large collections of atypical mitochondria, many with abnormal cristae (arrows). $M$, Myofibers; bar, $50 \mu \mathrm{m}$. c In addition, collections of rod structures can be seen in this electron micrograph, in which two are marked with arrows. The lipid droplet $(L)$ in the endomysium is a sign of degeneration. Bar $1 \mu \mathrm{m}$

Fig. 5. Cricopharyngeus muscle from patient 3 . The muscle fibers contain similar subsarcolemmal clumps as in Fig. 3a (arrows). In addition, fat and fibrous connective tissue has accumulated in the endomysium $(E)$, and is excessive even for the cricopharyngeal muscle (lower part of the figure). NADH-TR; bar, $100 \mu \mathrm{m}$ older subjects) [3]. The presence of these changes may depend on the exceptional function of the $\mathrm{CM}$ being contracted most of the time with only temporary relaxation during swallowing. This special function of the CM is also reflected in the predominance of the slow type 1 fibers. Additionally, the muscle is directly exposed to physical and chemical stresses through foods or liquids swallowed, as exemplified by hot beverages and strong alcohols. The large amount of connective tissue seen has also been described to the sphincter character and is needed for the circumferentially oriented myofibers to attach to. Against this background the changes in the 


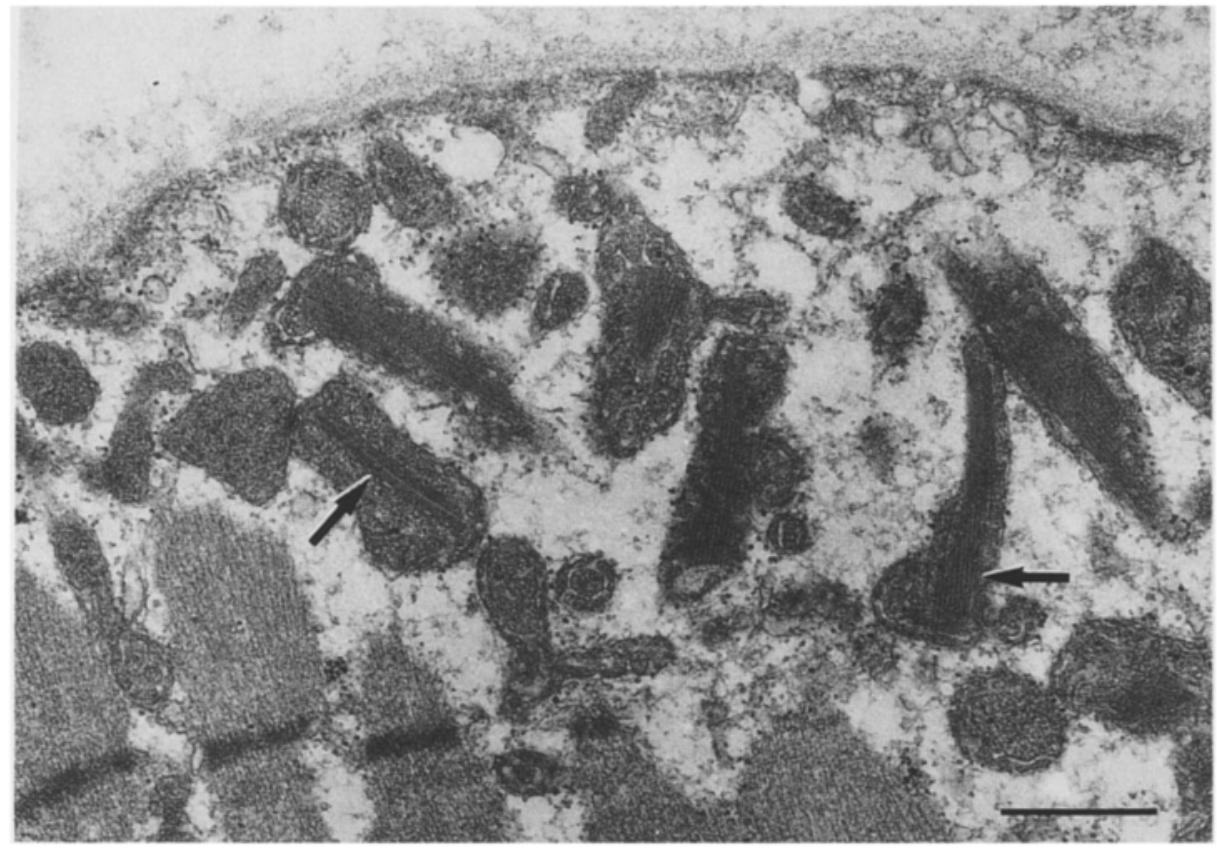

Fig. 6. An electron micrograph from the cricopharyngeus muscle of patient 4 .

The subsarcolemmal mitochondria contain abnormal paracrystalline inclusions (arrows). Bar, $0.5 \mu \mathrm{m}$
CMs of the dysphagia patients must be interpreted with caution (Dr. M. Mahon, personal communication).

All five of our patients with ICD seem to have different causes for their dysphagias. The definite inflammatory changes with fiber necrosis in patient 1 must be considered significant. Since small inflammatory infiltrates were also encountered in his vastus lateralis muscle, it is quite plausible that dysphagia was due to an inflammatory myopathy. This underlines the fact that myositis may manifest itself in the $\mathrm{CM}$ alone. A detailed neurologial examination with EMG and muscle biopsy is needed in these cases, since steroid therapy rather than surgery would be a treatment of choice. In our patient with inflammatory muscle changes a definite type of myositis could not be identified, i.e. EM specimens failed to show undulating tubules associated with dermatomyositis or filaments of inclusion body myositis. Since the patient was satisfied with his operative result and did not have other skeletal muscle disorders, steroid therapy was not considered necessary.

Patient 2 also had a few necrotic myofibers, inflammatory cells and nemaline rods in her CM fibers, as also previously described by Hanna and Henderson [13]. These rods represent a non-specific degenerative change, although they are abundant in a congenital nemaline myopathy [23] and represent its identifying stigma. Since neurological examination and vastus lateralis muscle biopsies were normal, there is no reason to ascribe this patient's ICD to a generalized myopathy. Furthermore, concurrent alcohol abuse could have contributed to the $\mathrm{CM}$ changes found.

In patient 3 the $\mathrm{CM}$ fibers were clearly larger than in the controls. This patient's age and undernourishement may explain the atrophy found in the deltoid type 2 fibers examined. Despite additional marked fibrosis and fat accumulation, the size of the patient's CM was clinically normal. In this case, the myofiber hypertrophy is most likely to be compensatory after fiber loss and the amount of reactive connective tissue seen $(65.4 \%)$ was excessive even for the $\mathrm{CM}$ and may well have contributed to dysphagia. Increased connective tissue was also present in patient $5(46.4 \%)$, but its significance is unclear.

Taken together, three of our five patients with ICD were found to have abnormal EM and immunohistochemistry changes. These changes reflected specific findings of myositis in one of the patients. Although we consider our control specimens to be reasonably reliable to provide information about the ultrastructure of the $\mathrm{CM}$ (when examined by EM), the size of our control group is small. As a consequence, the varying appearances of the $\mathrm{CM}$ in our specimens make it difficult to draw any firm conclusions.

In spite of the great variety of possible etiologies causing ICD in our patients, all benefited subjectively and objectively from CM myotomy. On the basis of present and previous studies $[2,11,18-20], \mathrm{CM}$ myotomy is generally a well-tolerated and safe operative treatment for idiopathic dysphagia. Moreover, in cases with pouch formation, myotomy together with resection of the diverticulum is considered to be the treatment of choice. Nonetheless, ICD mostly affects older patients who generally have increased operative risks. Thus, conservative treatment should be taken into account whenever possible. Further neurological and neurophysiological assessments should be performed before considering surgical care, even in patients with no specific signs of neurological disease.

\section{References}

1. Belsey R (1966) Functional disease of the esophagus. J Thorac Cardiovase Surg 52:164-188

2. Bonavina L, Khan NA, DeMeester TR (1985) Pharyngoesophageal dysfunctions. The role of cricopharyngeal myotomy. Arch Surg 120:541-549 
3. Bonington A, Mahon M, Whitmore I (1988) A histological and histochemical study of the cricopharyngeus muscle in man. J Anat 156:27-37

4. Collan Y, Salmenperä M (1976) Electron microscopy of postmortem autolysis of rat muscle tissue. Acta Neuropathol (Berl) $35: 219-233$

5. Cruse JP, Edwards DA, Smith JF, Wyllie JH (1979) The pathology of cricopharyngeal muscle. Histopathology 3:223-232

6. Delahunty JE, Margulies SI, Alonso WA, Kundson DH (1971) The relationship of reflux esophagitis to pharnygeal pouch (Zenker's diverticulum) formation. Laryngoscope 81:570-577

7. Dubowitz V (ed) (1985) Muscle biopsy: a practical approach. Bailliere Tindall, Philadelphia

8. Ekberg O (1986) The cricopharyngeus revisited Br J Radiol $59: 875-879$

9. Ekberg O, Lindgren S (1987) Effect of cricopharyngeal myotomy on pharyngoesophageal function: pre- and postoperative cineradiographic findings. Gastrointest Radiol 12:1-6

10. Ekberg O, Wahlgren L (1985) Dysfunction in pharyngeal swallowing. A cineradiographic investigation in 854 dysphagia patients. Acta Radiol Diagn (Stockh) 26:389-395

11. Ellis FH Jr, Crozier RE (1981) Cervical esophageal dysphagia: indications for and results of cricopharyngeal myotomy. Ann Surg 194:279-289

12. Ey W (1986) Zur operativen Therapie der Schlucklähmung. Laryngol Rhinol Otol 65:223-225

13. Hanna W, Henderson RD (1980) Nemaline rods in cricopharyngeal dysphagia. Am J Clin Pathol 74:186-191

14. Hendersson RD, Marryat GC (1977) Cricopharyngeal myotomy as a method of treating cricopharyngeal dysphagia secondary to gastroesophageal reflux. J Thorac Cardiovasc Surg $74: 721-725$
15. Holinshead WH (1968) Anatomy of surgeons, 2nd edn vol 1. The head and neck. Hoeber Medical Division, Harper and Row, New York, pp 456-462

16. Kaplan S (1951) Paralysis of deglutition, a post-poliomyelitis complication treated by section of the cricopharyngeus muscle. Ann Surg 133:572-573

17. Kristmunsdottir F, Mahon MMQ, Froes WJK (1990) Histomorphometric and histopathological study of the human cricopharyngeus muscle: in health and motor neuron disease. Neuropathol Appl Neurobiol 16:461-475

18. Lund WS (1968) The cricopharyngeal sphincter: its relationship to the relief of pharyngeal paralysis and the surgical treatment of the early pharyngeal pouch. J Laryngol Otol 82 : 353367

19. Mills CP (1973) Dysphagia in pharyngeal paralysis treated by cricopharyngeal sphincterotomy. Lancet $I: 455-457$

20. Pearson CM, Currie S (1974) Polymyositis and related disorders. In: Walton JN (ed) Disorders of voluntary muscles. Churchill-Livingstone, London, pp 614-652

21. Porubsky ES, Murray JP, Pratt LL (1973) Cricopharyngeal achalasia in dermatomyositis. Arch Otolaryngol 98:428-429

22. Seaman WB (1966) Cineradiographic observations of the cricopharyngeus. Am J Roentgenol Radium Ther Nucl Med 96: 922-931

23. Shy GM, Ergel WK, Somers JE, Wanko T (1963) Nemaline myopathy: a new congenital myopathy. Brain 86:793-810

24. Sorensen FB, Klebe JG, Henriques UV (1988) Intramuscular "mulberry-like" bodies: morphological, immunohistochemical, and ultrastructural observations of an incidental finding caused by autolysis? Pathol Res Pract 183:88-94

25. Wilkins SA JR (1964) Indications for section of the cricopharyngeus muscle. Am J Surg 108:533-538 